\title{
Chung-Feller property of Schröder objects
}

\author{
Youngja Park \\ Department of Mathematics \\ Yonsei University \\ Seoul 120-749, Republic of Korea
}

ojpark@yonsei.ac.kr

\author{
Sangwook Kim \\ Department of Mathematics \\ Chonnam National University \\ Gwangju 500-757, Republic of Korea \\ swkim.math@chonnam.ac.kr
}

Submitted: Oct 26, 2015; Accepted: May 1, 2016; Published: May 13, 2016

Mathematics Subject Classifications: 05A15; 05A19

\begin{abstract}
Large Schröder paths, sparse noncrossing partitions, partial horizontal strips, and 132-avoiding alternating sign matrices are objects enumerated by Schröder numbers. In this paper we give formula for the number of Schröder objects with given type and number of connected components. The proofs are bijective using ChungFeller style. A bijective proof for the number of Schröder objects with given type is provided. We also give a combinatorial interpretation for the number of small Schröder paths.
\end{abstract}

Keywords: large and small Schröder paths; partial horizontal strips; sparse noncrossing partitions; 132-avoiding alternating sign matrices

\section{Introduction}

A (large) Schröder path of length $2 n$ is a lattice path from $(0,0)$ to $(2 n, 0)$ using up steps $U=(1,1)$, flat steps $F=(2,0)$, and down steps $D=(1,-1)$ such that it stays weakly above the $x$-axis. For example, the path $U D F U F U D U F D D$ shown in Figure 1(a) is a large Schröder path of length 14 . We denote the set of large Schröder paths of length $2 n$ by $\mathcal{R}_{n}$ and call $r_{n}:=\left|\mathcal{R}_{n}\right|$ a Schröder number. It is known [14] that the $n$-th Schröder number is $r_{n}=\frac{1}{n} \sum_{k=1}^{n}\left(\begin{array}{c}n \\ k-1\end{array}\right)\left(\begin{array}{l}n \\ k\end{array}\right) 2^{k}$ and the initial terms are $\left\{r_{n}\right\}_{n \geqslant 0}=\{1,2,6,22,90,384, \ldots\}_{n \geqslant 0}$. A descent is a maximal sequence of (consecutive) $D$ steps and the (descent) type of a large Schröder path is the integer partition formed by the length of the descents. For example, the large Schröder path UDFUFUDUFDD has type $\lambda=(2,1,1)$. A small Schröder path is a large Schröder path with no flat steps on the $x$-axis.

A Dyck path of length $2 n$ is a lattice path from $(0,0)$ to $(2 n, 0)$ using up steps $U$ and down steps $D$ such that it stays weakly above the $x$-axis. We denote the set of Dyck paths of length $2 n$ by $\mathcal{D}_{n}$. It is well known [14] that the cardinality $\left|\mathcal{D}_{n}\right|$ is the Catalan numbers $c_{n}:=\frac{1}{n+1}\left(\begin{array}{c}2 n \\ n\end{array}\right)$, with initial terms $\left\{c_{n}\right\}_{n \geqslant 0}=\{1,1,2,5,14,42,132, \ldots\}_{n \geqslant 0}$. The 
type of a Dyck path can be defined by considering it as a large Schröder path without flat steps. A Dyck path is said to be connected if it doesn't touch the $x$-axis except two end points. Note that a small Schröder path may not be a connected Dyck path since a small Schröder paths may touch the $x$-axis more than twice.

The Dyck paths and the large Schröder paths are closely related and both are fundamental families of lattice paths. Hence it is natural to extend results on Dyck paths to large Schröder paths (or vice versa). The number of Dyck path of given type is known since Kreweras [9] introduced noncrossing partitions while the number of large Schröder path of given type is recently given by $\mathrm{An}, \mathrm{Eu}$, and Kim [1]. Rhoades [13] enumerates the number of Dyck paths of fixed type and fixed number of returns to the $x$-axis. In this article, we count large Schröder paths which have a fixed number of flat steps on the $x$-axis according to type. We also extend these results to Motzkin and Riordan paths.

The classical Chung-Feller theorem [4] provides an elegant way to enumerate the number of Dyck path. It says that the number of free Dyck paths with $k$ flaws is independent of $k$. The classical Chung-Feller theorem was first proved by MacMahon [11] and Chung and Feller reproved by analytic method [4]. Narayana [12] gave a combinatorial proof by using cyclic permutations of paths and Chen [3] gave a bijective proof.

There are several results about Chung-Feller property of large Schröder paths. Eu, Fu, and Yeh [5] give weighted Chung-Feller theorem for large Schröder paths. Chen, Li, and Shapiro [2] give butterfly decomposition which implies Eu et al's therem about weighted large Schröder paths. Liu, Wang, and Yeh [10] provide Chung-Feller type results about large Schröder paths.

Huq [6] applied the "cycle method" to develop generalized Chung-Feller theorems. He gave nice combinatorial interpretations to the Catalan number formula as well as the Narayana number formula. He also discuss the number of Motzkin and large Schröder paths. We provide a combinatorial interpretation for the number of Riordan and small Schröder paths.

The rest of this paper is organized as follows. In Section 2, we list several objects counted by Schröder numbers. We provide enumeration results in Section 3. The proof of the main theorem is given in Section 4. In the last section, we provide a new proof for the number of large Schröder paths with a given type and give a combinatorial interpretation for the number of small Schröder paths.

\section{$2 \quad$ Schröder objects}

In this section, we introduce several objects counted by Schröder numbers. We focus on Schröder objects which have natural notions of type and connectedness.

\subsection{Sparse noncrossing partitions}

A partition $\pi$ of a set $[n]:=\{1,2, \ldots, n\}$ is a collection $B_{1}, B_{2}, \ldots, B_{\ell}$ of nonempty pairwise disjoint subsets of $[n]$ whose union is $[n]$. The $B_{i}$ 's are called blocks. The type of a noncrossing partition is the integer partition given by its block sizes. The 


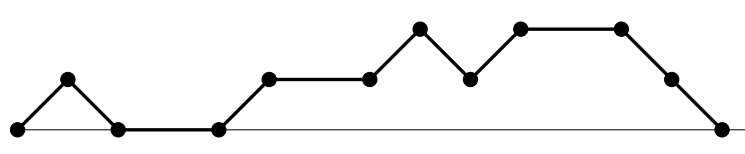

(a) A large Schröder path $P$

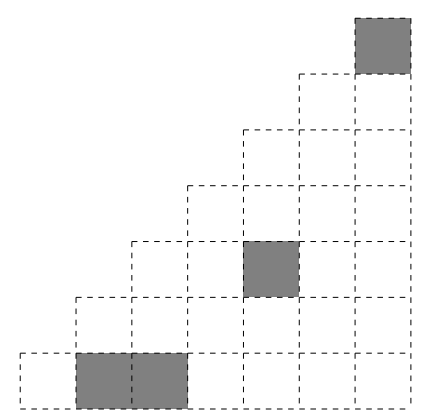

(c) A partial horizontal strip $\sigma$

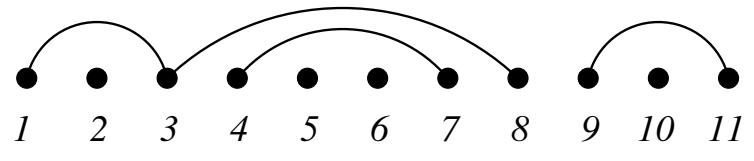

(b) A Sparse noncrossing partition $\pi$

Figure 1: Schröder objects

standard representation of a partition $\pi$ is a graph on the vertex set $[n]$ such that a block $B=\left\{b_{1}, b_{2}, \ldots, b_{k}\right\}$ corresponds to a path $\left(b_{1}, b_{2}, \ldots, b_{k}\right)$. A pair $(i, j)$ with $i<j$ which corresponds to an edge in the standard representation of $\pi$ is called an arc. The arc type of a partition $\pi$ is the integer partition given by length of paths in the standard representation of $\pi$. If the type of a partition $\pi$ is $\left(\lambda_{1}, \lambda_{2}, \ldots, \lambda_{\ell}, 1, \ldots, 1\right)$ with $\lambda_{\ell} \geqslant 2$, then its arc type is $\left(\lambda_{1}-1, \lambda_{2}-1, \ldots, \lambda_{\ell}-1\right)$. For a noncrossing partition $\pi=(\{1,3,8\},\{2\},\{4,7\},\{5\},\{6\},\{9,11\},\{10\})$ shown in Figure 1(b), its type is $(3,2,2,1,1,1,1)$ and its arc type is $(2,1,1)$.

The set partition is called connected if for every $i$ with $1 \leqslant i<n$ there exists at least one arc in the standard representation which connects the intervals $[1, i]$ and $[i+1, n]$. The set partition is said to have $m$ connected components if there exist numbers $1 \leqslant$ $i_{1}<i_{2}<\cdots<i_{m-1}<n$ such that the restriction of the its standard representation to each of the intervals $\left[1, i_{1}\right],\left[i_{1}+1, i_{2}\right], \ldots,\left[i_{m-1}+1, n\right]$ is a connected set partition. A set partition is called sparse if no two consecutive integers are in the same block. A partition is noncrossing if $a, c \in B_{i}$ and $b, d \in B_{j}$ for no elements $a<b<c<d$ and $i \neq j$. The partition $\pi$ listed above is sparse, noncrossing and has 2 connected components. It is well-known that the sparse noncrossing partitions of some set into $n$ blocks are counted by large Schröder numbers $r_{n-1}$. (See [8] or [14, Exercise 6.39.o].)

\subsection{Partial horizontal strips}

Let $\mu=\left(\mu_{1}, \mu_{2}, \ldots\right)$ and $\nu=\left(\nu_{1}, \nu_{2}, \ldots\right)$ be integer partitions such that $\mu_{i} \geqslant \nu_{i}$ for all $i$. The skew shape $\mu / \nu$ is the setwise difference of Young diagrams. A partial horizontal strip in the shape $\mu / \nu$ is a set of boxes, at most one in each column, such that the height of the boxes is weakly increasing to the right. The collection of numbers of boxes in each 
row of a partial horizontal strip $\sigma$ form an integer partition, called the type of $\sigma$. The type of the partial horizontal strip $\sigma$ shown in Figure $1(\mathrm{c})$ is $(2,1,1)$.

In this article, we are interested in partial horizontal strips contained in the staircase skew shape $n^{n} /(n-1, \ldots, 2,1)$. The partial horizontal strips in the staircase skew shape is counted by Schröder numbers [1]. A partial horizontal strip in the staircase skew shape is connected if there is no empty box in the main diagonal such that all the chosen boxes are either strictly above the corresponding row or strictly to the left of the corresponding column. If there are $m-1$ such empty boxes, then the partial horizontal strip is said to have $m$ connected components. The partial horizontal strip $\sigma$ shown in Figure 1(c) has 2 connected components since the leftmost empty box in the second row from the top is the unique empty box which satisfies the above condition.

\section{$2.3 \quad 132$-avoiding alternating sign matrices}

An alternating sign matrix is a matrix consisting of $0 \mathrm{~s}, 1 \mathrm{~s}$, and $-1 \mathrm{~s}$ where the sum of entries in each row and in each column is 1 and the signs of nonzero entries of each row and of each column are alternating. For an $n \times n$ alternating sign matrix $M$ and a permutation $\pi$, we say that $M$ contains $\pi$ if $M$ contains a submatrix $D=\left(d_{i j}\right)$ where $d_{i j}=1$ whenever $\pi(i)=j$. If $M$ does not contain $\pi$, we say that $M$ avoids $\pi$. Johansson and Linusson [7] show that the 132-avoiding alternating sign matrices are counted by the Schröder numbers.

An 132-avoiding alternating matrix $M$ is connected if there is only one 1 which is the unique nonzero entry in its row and all the entries in its lower right positions are zero. If there are $m$ such 1 's, then we say that $M$ has $m$ connected components. The matrix $M$ shown in Figure 1(d) has two connected components since the 1's on the 6th row and the 8th row satisfy the above condition.

Alternating sign matrices carry a notion of type defined as follows: Circle all 1's such that all the entries which lie northeast of it are 0 and connect them with a path from top to bottom. Circle all 1's such that all the entries which lie northeast (including north or east) of it are $0,-1$ or circled 1 and connect them with a path from top to bottom. Continue this until all 1's are circled. The type of an 132-avoiding alternating matrix is the integer partition given by the length of paths. The type of the matrix $M$ shown in Figure $1(\mathrm{~d})$ is $(2,1,1)$.

\section{Enumeration of Schröder objects by type and connected com- ponents}

In this section, we enumerate the number of Schröder objects by type and number of connected components.

First, we define several notions which will be used throughout this paper. The height of a path $P$ is the height of its lowest point and is denoted by $h(P)$. The height of a subpath or a step is similarly defined. A negative step is a step whose height is negative. Positive, nonnegative, and nonpositive steps are defined similarly. For an up step $U$, its 
matching down step is the first down step after $U$ whose height is the same as $U$. An up step is the matching up step of its matching down step. The length of a descent $\bar{D}$ is the number of its down steps and is denoted by $\ell(\bar{D})$.

Given an integer partition $\lambda$, we set $m_{\lambda}:=m_{1}(\lambda) ! m_{2}(\lambda) ! m_{3}(\lambda) ! \cdots$, where $m_{i}(\lambda)$ is the number of parts of $\lambda$ equal to $i$. Note that $m_{\lambda}$ here is not the monomial symmetric function. We use $|\lambda|$ for the sum of the parts of $\lambda$.

Before we state the main theorem, we give a lemma about two statistics of large Schröder paths which are equi-distributed.

Lemma 1. Among all large Schröder paths of given length and type, the number of flat steps on the $x$-axis and the number of flat steps after the last up step are equi-distributed.

Proof. Let $P$ be a large Schröder path of given length and type. We provide an algorithm which sends $P$ to $Q$ so that all the flat steps of height 0 of $P$ are sent to the flat steps after the last up step of $Q$ and vice versa.

Decompose $P$ into $P=\tau_{1} \sigma_{1} \tau_{2} \sigma_{2} \cdots \tau_{j} \tau_{j}^{\prime} \sigma_{j} \cdots \tau_{k} \sigma_{k} \alpha \beta \bar{D}$ where

- $\bar{D}$ is the last descent,

- $\sigma_{i}$ is the $i$ th flat step of height 0 together with a descent immediate before it (if any),

- if $1 \leqslant i \leqslant k$ and $\sigma_{i}$ is not the last one with a descent, then $\tau_{i}$ is the subpath between $\sigma_{i-1}$ and $\sigma_{i}$ (if $\sigma_{i}$ does not have a descent, $\tau_{i}$ is empty),

- if $\sigma_{j}$ is the last one with a descent, then $\tau_{j}$ is the subpath after $\sigma_{j-1}$ up to the matching up step of the first down step of $\sigma_{j}$ and $\tau_{j}^{\prime}$ is the subpath between $\tau_{j}$ and $\sigma_{j}\left(\tau_{j}^{\prime}\right.$ could be empty and it ends with $F$ if it is not empty),

- $\alpha$ is the subpath after $\sigma_{k}$ up to the matching up step of the first down step of $\bar{D}$,

- $\beta$ is the subpath between $\alpha$ and $\bar{D}$ (the height of $\beta$ is $\ell(\bar{D})$ and $\beta$ must finish with $F$ unless it is empty.).

Then let $Q:=\beta \alpha \tau_{j}^{\prime} \tau_{1} \tau_{2} \cdots \tau_{k} \sigma_{1} \sigma_{2} \cdots \sigma_{k} \bar{D}$. Note that all the flat steps of height 0 are moved to the flat steps after the last up step.
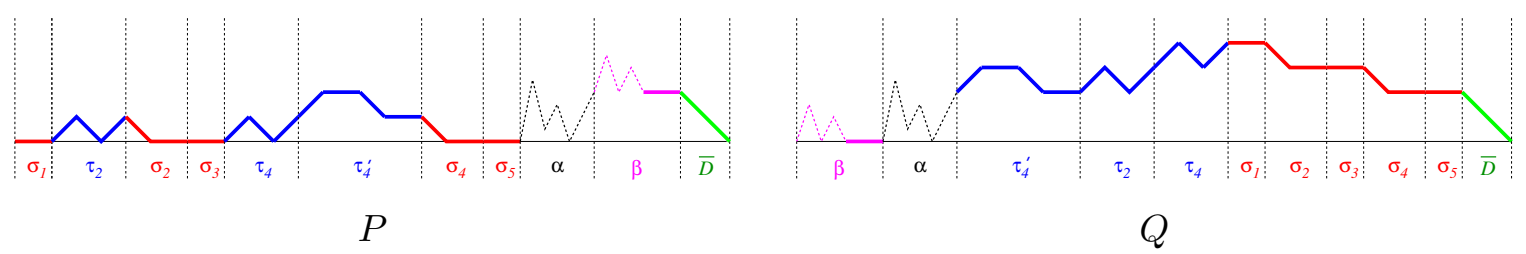

Figure 2: Algorithm for sending flat steps of height 0 to flat steps after the last up

Conversely, decompose $Q$ into $Q=\beta \alpha \tau_{j}^{\prime} \tau_{1} \tau_{2} \cdots \tau_{k} \sigma_{1} \sigma_{2} \cdots \sigma_{k} \bar{D}$, where 
- $\bar{D}$ is the last descent,

- $\beta$ is the subpath from the beginning to the last flat step of height 0 ,

- $\alpha$ is the subpath after $\beta$ up to the matching up step of the first down step of $\bar{D}$,

- $\sigma_{i}$ : the $i$ th flat step after the last up step together with a descent immediate before it (if any),

- if $\sigma_{j}$ is the last one with a descent, $\tau_{j}^{\prime}$ is the subpath after $\alpha$ up to the last flat step of height $\ell(\bar{D})$ before the last up step (if no such flat steps exist, $\tau_{j}^{\prime}$ is empty),

- For $i<j, \tau_{i}$ is the subpath after $\tau_{i-1}$ up to the last $U$ or $F$ ending at height $\ell(\bar{D})+\ell_{1}+\cdots+\ell_{i}$ where $\ell_{i}$ is the length of the descent in $\sigma_{i}$. (if $\ell_{i}=0$, then $\tau_{i}$ is empty.) $\tau_{j}$ is the subpath after $\tau_{j-1}$ up to the last $U$. For $i>j, \tau_{i}$ is empty.

Then let $P:=\tau_{1} \sigma_{1} \tau_{2} \sigma_{2} \cdots \tau_{j} \tau_{j}^{\prime} \sigma_{j} \cdots \tau_{k} \sigma_{k} \alpha \beta \bar{D}$. Note that all the flat steps after the last up step are moved to the flat steps of height 0 . Also, it is easy to see that these maps are inverse to each other.

The following is the main theorem of this article which provides the number of Schröder objects of given length and type with a fixed number of connected components.

Theorem 2. The number of

1. large Schröder paths of length $2 n$ with type $\lambda=\left(\lambda_{1}, \ldots, \lambda_{\ell}\right)$ with $m-1$ flat steps on the $x$-axis

2. large Schröder paths of length $2 n$ with type $\lambda$ with $m-1$ flat steps after the last up step

3. sparse noncrossing set partitions of $[n+|\lambda|+1]$ with arc type $\lambda$ and $m$ connected components

4. partial horizontal strips with type $\lambda$ and $m$ connected components contained in the staircase skew shape $n^{n} /(n-1, \ldots, 2,1)$

5. 132-avoiding $(n+1) \times(n+1)$ alternating sign matrices with type $\lambda$ and $m$ connected components

is given by

$$
\frac{m}{n+1}\left(\begin{array}{c}
n-m \\
|\lambda|-1
\end{array}\right)\left(\begin{array}{c}
n+1 \\
\ell
\end{array}\right) \frac{\ell !}{m_{\lambda}}
$$

The equivalence of Part 1 and 2 follows from Lemma 1. The equivalence of Part 1, 3, and 4 can be obtained from the bijections provided by An, Eu, and Kim [1]. The bijection given by Johansson and Linusson [7] implies the equivalence of Part 1 and 5. The proof of Theorem 2 is given in Section 4 . 
A small Schröder path is a large Schröder path with no flat steps on the $x$-axis. It is well-known that the number of small Schröder paths of length $2 n$ is the half of the number of large Schröder paths of length $2 n$. This is not the case when we count the number of small Schröder paths of fixed type. The following corollary provides the number of small Schröder paths of given type.

Corollary 3. The number of

1. small Schröder paths of length $2 n$ with type $\lambda=\left(\lambda_{1}, \ldots, \lambda_{\ell}\right)$

2. large Schröder paths of length $2 n$ with type $\lambda$ with no flat steps after the last up step

3. connected sparse noncrossing set partitions of $[n+|\lambda|+1]$ with arc type $\lambda$

4. connected partial horizontal strips with type $\lambda$ contained in the staircase skew shape $n^{n} /(n-1, \ldots, 2,1)$

5. connected 132-avoiding $(n+1) \times(n+1)$ alternating sign matrices with type $\lambda$ is given by

$$
\frac{1}{n+1}\left(\begin{array}{c}
n-1 \\
|\lambda|-1
\end{array}\right)\left(\begin{array}{c}
n+1 \\
\ell
\end{array}\right) \frac{\ell !}{m_{\lambda}}
$$

A Motzkin path of length $n$ is a lattice path from $(0,0)$ to $(n, 0)$ using up steps $(1,1)$, flat steps $(1,0)$, and down steps $(1,-1)$ such that it stays weakly above the $x$-axis. A Riordan path is a Motzkin path with no flat steps on the $x$-axis. As the flat steps do not affect the type of a path, there is a bijection between the set of Motzkin paths of length $n$ with type $\lambda$ and the set of large Schröder paths of length $2(n-|\lambda|)$ with type $\lambda$. Similar bijection can be obtained between Riordan paths and small Schröder paths. Thus we get the following corollaries.

Corollary 4. The number of Motzkin paths of length $n$ with type $\lambda$ with $m-1$ flat steps on the $x$-axis is

$$
m\left(\begin{array}{c}
n-|\lambda|-m \\
|\lambda|-1
\end{array}\right)\left(\begin{array}{c}
n-|\lambda| \\
\ell-1
\end{array}\right) \frac{(\ell-1) !}{m_{\lambda}} .
$$

Corollary 5. The number of Riordan paths of length $n$ with type $\lambda=\left(\lambda_{1}, \ldots, \lambda_{\ell}\right)$ is

$$
\left(\begin{array}{c}
n-|\lambda|-1 \\
|\lambda|-1
\end{array}\right)\left(\begin{array}{c}
n-|\lambda| \\
\ell-1
\end{array}\right) \frac{(\ell-1) !}{m_{\lambda}}
$$

\section{Proof of the main theorem}

In this section, we provide an algorithm which shows that the number of paths is independent of the number of flaws.

The type of a lattice path is the integer partition formed by the length of the descents. Let $L(n, \lambda, m)$ be the set of all lattice paths of type $\lambda$ from $(0,0)$ to $(2 n+1,1)$ using 
$U, F$, or $D$ beginning with up or flat step and containing $m-1$ flat steps before the first up step or after the last up step. The number of flaws of a path $P \in L(n, \lambda, m)$ is the number of nonpositive flat steps and nonpositive up steps. Let $L_{k}(n, \lambda, m)$ be the subset of $L(n, \lambda, m)$ consisting of lattice paths with $k$ flaws $(1 \leqslant k \leqslant n+1)$. We give a bijection between $L_{k}(n, \lambda, m)$ and $L_{k-1}(n, \lambda, m)$ when $k \geqslant 2$.

\subsection{Algorithm for the subpath after the last up step of height 0}

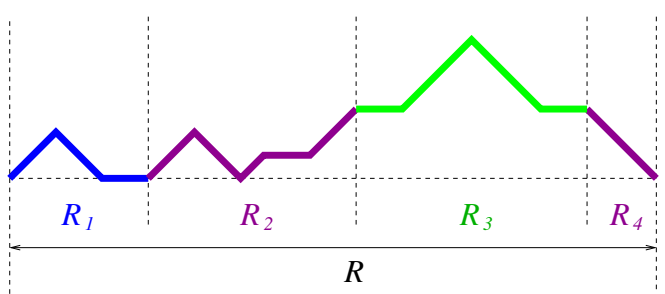

$R$
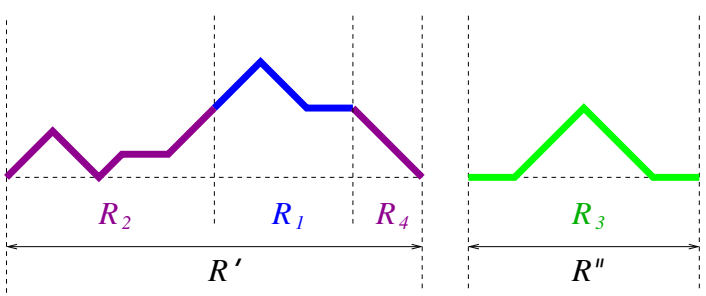

$\left(R^{\prime}, R^{\prime \prime}\right)$

Figure 3: Algorithm $R \leftrightarrow\left(R^{\prime}, R^{\prime \prime}\right)$

Let $P$ be a path in $L_{k}(n, \lambda, m)$ and let $R$ be the subpath of $P$ after the last up step of height 0 . If $R$ ends with $D$, decompose $R$ into $R_{1} R_{2} R_{3} R_{4}$ where

- $R_{1}$ : the part of $R$ from the beginning to the last flat step of height 0 (if there is no flat step of height $0, R_{1}$ is empty),

- $R_{4}$ : the last descent of $R$,

- $R_{2}$ : the subpath after $R_{1}$ up to the matching up step of the first down step of $R_{4}$,

- $R_{3}$ : the subpath between $R_{2}$ and $R_{4}$.

Let $R^{\prime}:=R_{2} R_{1} R_{4}$ and $R^{\prime \prime}:=R_{3}$. If $R$ ends with $F$, then $R^{\prime}$ is the empty path and $R^{\prime \prime}=R$. Note that $R^{\prime}$ has no flat step on its height.

Conversely, suppose two paths $R^{\prime}$ and $R^{\prime \prime}$ are given where $R^{\prime}$ is a path whose height is the height of the first step with no flat step on its height. If $R^{\prime}$ is empty, let $R=R^{\prime \prime}$. Otherwise, $R^{\prime}$ ends with a descent. Decompose $R^{\prime}=R_{2} R_{1} R_{4}$ where $R_{4}$ is the last descent, $R_{2}$ is the subpath from the beginning to the matching up step of the first down step of $R_{4}$, and $R_{1}$ is the subpath between $R_{2}$ and $R_{4}$. Let $R:=R_{1} R_{2} R^{\prime \prime} R_{4}$.

\subsection{Algorithm for decreasing the number of flaws}

Now, we describe the main algorithm. There are several cases:

1. When there are more than one up steps of height 0 and $R$ is not following $D U$ : There are three sub cases: 
(a) $R$ is following $F U$ :

If there is a down step of height 0 whose previous step is either a flat step or a down step, then let $Q$ be the subpath from the descent containing the last such down step to the preceding step of $F U$. Otherwise, let $Q$ be the path from the next step of the first up step of height 0 to the preceding step of $F U$. Decompose $P$ into $S Q F U R$. Construct the path $\phi(P):=S R^{\prime} F Q U R^{\prime \prime}$.
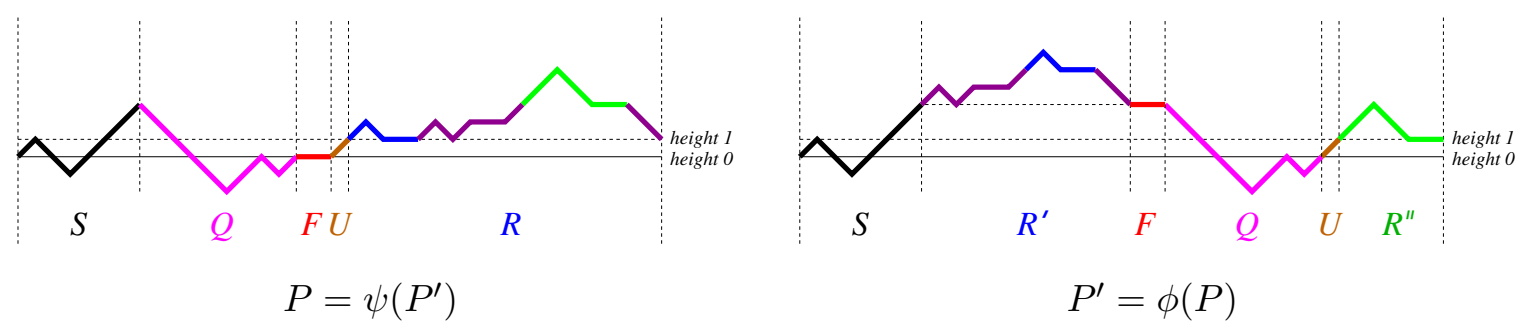

Figure 4: Main algorithm, Case 1(a)

(b) $R$ is following $U U$ and there is no flat step of height 0 after the last down step of height 0 :

Let $Q$ be the part of the path from the last descent beginning on positive height to the last up step of height -1 . Decompose $P$ into $S Q U R$. Construct the path $\phi(P):=S R^{\prime} U Q R^{\prime \prime}$.
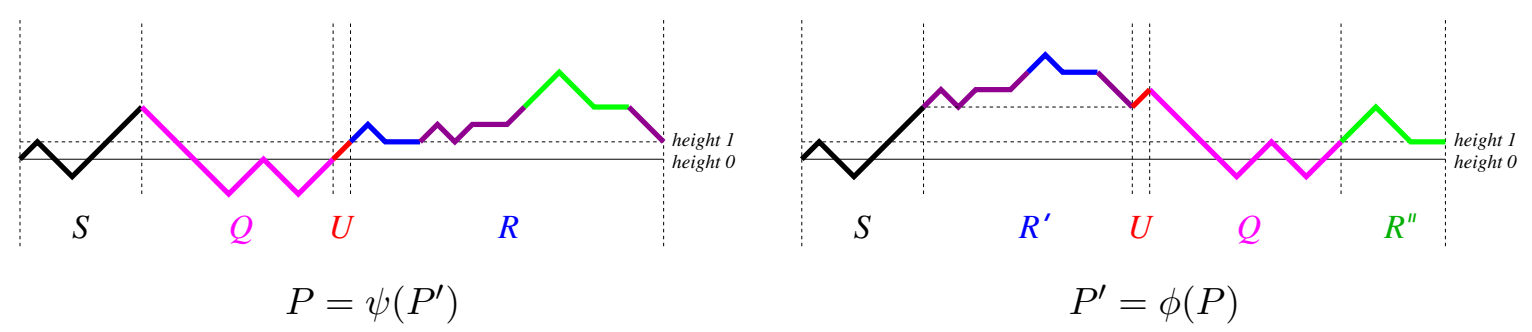

Figure 5: Main algorithm, Case 1(b)

(c) $R$ is following $U U$ and there is a flat step of height 0 after the last down step of height 0 :

Let $F$ be the last such step and decompose $P$ into $S F Q U R$. Construct the path $\phi(P):=S U R^{\prime} F Q R^{\prime \prime}$.

2. When there are more than one up step of height 0 and $R$ is following $D U$ :

In this case, we can decompose the path into $S \bar{D} U R$. Construct a path $S R^{\prime} U R^{\prime \prime} \bar{D}$.

3. When there is only one up step of height 0 :

In this case, the path must begin with a flat step since $P$ contains at least two flaws. 

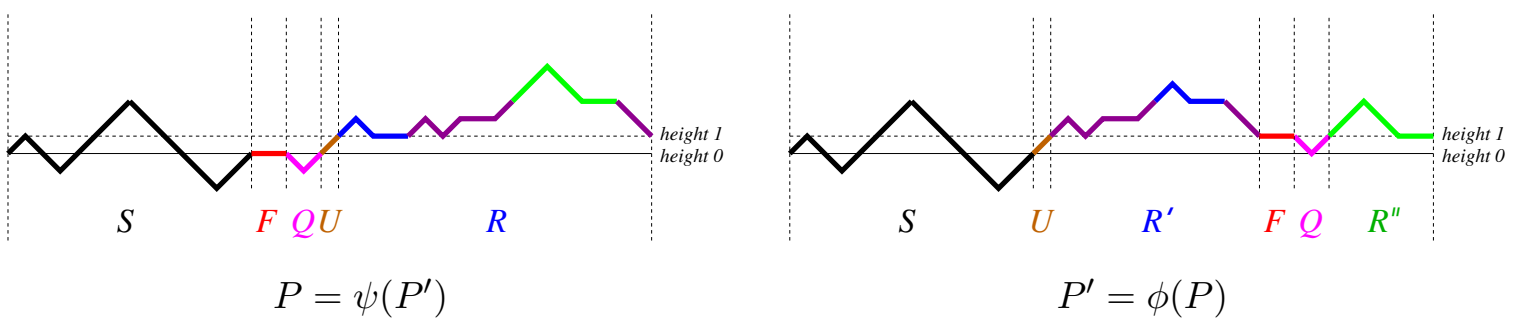

Figure 6: Main algorithm, Case 1(c)
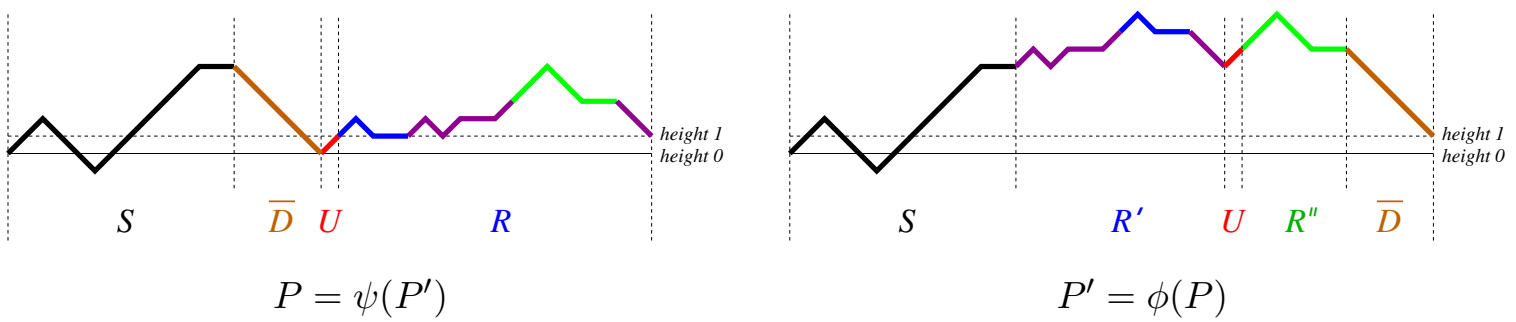

Figure 7: Main algorithm, Case 2

Let $\bar{D}$ be the descent preceding the first up step. (Note that $\bar{D}$ is following a flat step $F$ and it could be empty.) Decompose $P$ into $S F \bar{D} Q_{1} Q_{2} R$ where $Q_{2}$ is the subpath from the first up or flat step of height $1-\ell(\bar{D})$ to the unique up step of height 0 . Then $\phi(P)$ is the path $S Q_{1} \bar{D} Q_{2} R F$. Note that $R$ is not changed in this case.
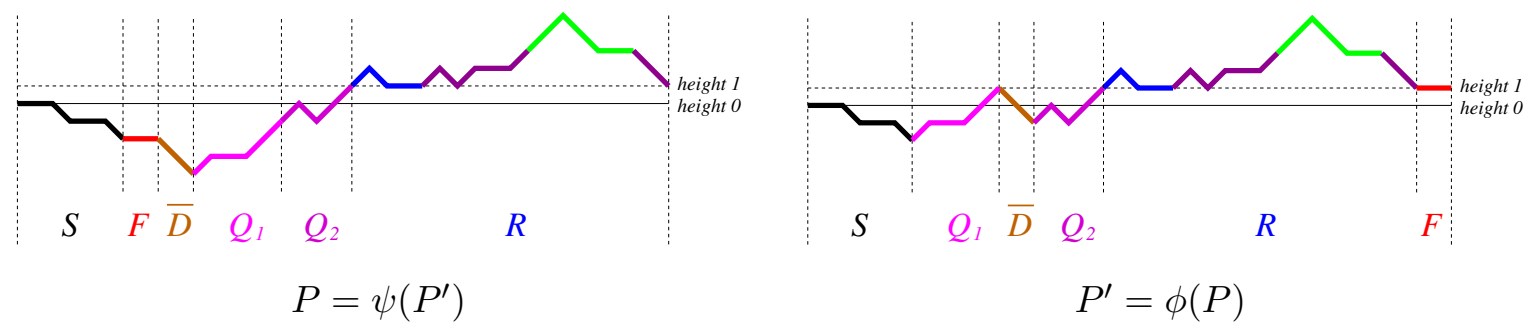

Figure 8: Main algorithm, Case 3

One can see that a path in $L_{k}(n, \lambda, m)$ must satisfy exactly one of the given cases and any two paths in different cases are not sent to the same case.

In order to decrease the number of flaws while keeping the number of flat steps before the first up step or after the last up step, a $U$ or $F$ of nonpositive height must be moved to the positive height. In Case 3, the last flat step before the first up step is moved. In Case 1(a) and 1(c), the last flat step of height 0 is moved. In Case 1(b) and 2, the last up step of height 0 is moved. 
In order to have a bijection, we must keep track of the position of the step moved. In Case 3, $\bar{D}$ is used for this purpose while $R^{\prime}$ is used in other cases.

We sent $R$ to $\left(R^{\prime}, R^{\prime \prime}\right)$ in order to keep the flat steps after the last up step. No two paths in the same case are sent to the same path since $R \leftrightarrow\left(R^{\prime}, R^{\prime \prime}\right)$ is a bijection and all the remaining steps except the moved ones $\left(U, F, \bar{D}, R^{\prime}\right)$ are fixed.

\subsection{Algorithm for increasing the number of flaws}

Let $P^{\prime}$ be a path in $L_{k-1}(n, \lambda, m)$. There are several cases and we list them according to the labels and order used in Subsection 4.2. The algorithm constructing $R$ from $R^{\prime}$ and $R^{\prime \prime}$ is discussed in Subsection 4.1.

1. When the path ends with $U$ or ends with $F$ and there is a subpath whose height is at least 1 before the last up step of height 0 :

(a) If it is not (b) nor (c):

Decompose $P^{\prime}$ into $S R^{\prime} F Q U R^{\prime \prime}$ where

- $U$ : the last up step of height 0

- $F$ : the last flat step of positive height before the above $U$

- $R^{\prime}$ : the largest connected subpath of height $h(F)$ immediate before the above $F$ with no flat steps of height $h(F)$

Let $\psi\left(P^{\prime}\right):=S Q F U R$.

(b) If the last nondecreasing step of positive height before the last $U$ of height 0 is $U$ :

Decompose $P^{\prime}=S R^{\prime} U Q R^{\prime \prime}$ where

- $R^{\prime \prime}$ : after the last up step of height 0 (could be empty)

- $U$ : the last nondecreasing step of positive height (the above $U$ )

- $R^{\prime}$ : the largest connected subpath of height $\geqslant h(F)$ immediate before the above $U$ with no $F$ on height $h(F)$

Let $\psi\left(P^{\prime}\right):=S Q U R$.

(c) If the last nondecreasing step of positive height before the last $U$ of height 0 is $F$ of height 1 and there are more than one $U$ of height 0 before that $F$, and there is no $F$ of height 1 between the last such $U$ and $F$ :

Decompose $P^{\prime}=S U R^{\prime} F Q R^{\prime \prime}$ where

- $R^{\prime \prime}$ : after the last up step of height 0 (could be empty)

- $F$ : the last $F$ of height 1 before the last $U$ (the above $F$ )

- $U$ : the last $U$ of height 0 before $F$ (the above $U$ )

Let $\psi\left(P^{\prime}\right):=S F Q U R$.

2. When the path ends with $D$ :

Decompose $P^{\prime}=S R^{\prime} U R^{\prime \prime} \bar{D}$ where 
- $\bar{D}$ : the last descent

- $U$ : the last up step of height $\ell(\bar{D})$

- $R^{\prime}$ : the largest connected subpath of height $\geqslant \ell(\bar{D})$ immediate before the above $U$ with no $F$ on height $\ell(\bar{D})$

Let $\psi\left(P^{\prime}\right):=S \bar{D} U R$.

3. When the path ends with $F$ and there is no subpath of positive height before the last up step of height 0 :

Decompose $P^{\prime}=S Q_{1} \bar{D} Q_{2} R F$ where

- $F$ : the last flat step

- $R$ : the subpath after the last up step of height 0 and before the above $F$

- $\bar{D}$ : the last descent beginning at height 1 (might be empty)

- $Q_{1}$ : the subpath from the first up step to the step immediate before $\bar{D}$

Let $\psi\left(P^{\prime}\right):=S F \bar{D} Q_{1} Q_{2} R$.

Now we provide the proof of the main theorem.

Proof of Theorem 2. Any path in $L(n, \lambda, m)$ contains $|\lambda|+1$ up steps, $n-|\lambda|$ flat steps, and $\ell$ descents. Since there are $m$ ways to put $m-1$ flat steps before the first up step and after the last up step, $\left(\begin{array}{c}n-m \\ |\lambda|-1\end{array}\right)$ ways to arrange the remaining $|\lambda|-1$ up steps and $n-|\lambda|-m+1$ flat steps between the first and the last up step, and $\left(\begin{array}{c}n+1 \\ \ell\end{array}\right) \frac{\ell !}{m_{\lambda}}$ ways to arrange $\ell$ descents, the number of paths in $L(n, \lambda, m)$ is

$$
m\left(\begin{array}{c}
n-m \\
|\lambda|-1
\end{array}\right)\left(\begin{array}{c}
n+1 \\
\ell
\end{array}\right) \frac{\ell !}{m_{\lambda}} .
$$

The number of flaws can vary from 1 to $n+1$. By the algorithms described, the number of paths is independent of the number of flaws. Since the lattice paths in $L_{1}(n, \lambda, m)$ begin with an up step and stay strictly above the $x$-axis except the origin, there is a one-to-one correspondence between $L_{1}(n, \lambda, m)$ and the set of all large Schröder paths of length $n$ with type $\lambda$ having $m-1$ flat steps after the last up step. Thus the theorem follows.

\section{$5 \quad$ Small and large Schröder paths}

In this section, we provide a new proof for the number of large Schröder paths with given type (and any number of connected components). Also, we give a combinatorial interpretation for the number of small Schröder paths (with any type).

An, Eu and Kim [1] show the following theorem about the number of large Schröder paths of given type. We provide a new bijective proof.

Theorem 6. The number of 
1. large Schröder paths of length $2 n$ with type $\lambda=\left(\lambda_{1}, \ldots, \lambda_{\ell}\right)$

2. sparse noncrossing set partitions of $[n+|\lambda|+1]$ with arc type $\lambda$

3. partial horizontal strips with type $\lambda$ contained in the staircase skew shape $n^{n} /(n-$ $1, \ldots, 2,1)$

4. 132-avoiding $(n+1) \times(n+1)$ alternating sign matrices with type $\lambda$

is

$$
\frac{1}{|\lambda|+1}\left(\begin{array}{c}
n \\
|\lambda|
\end{array}\right)\left(\begin{array}{c}
n+1 \\
\ell
\end{array}\right) \frac{\ell !}{m_{\lambda}} .
$$

Proof. Consider the set $L$ of all lattice paths from $(0,0)$ to $(2 n, 0)$ of type $\lambda$ using steps $U, F$, and $D$. Note that there are $|\lambda|$ up steps, $n-|\lambda|$ flat steps, and $\ell$ descents. Since there are $\left(\begin{array}{c}n \\ |\lambda|\end{array}\right)$ ways to arrange up steps and flat steps and there are $\left(\begin{array}{c}n+1 \\ \ell\end{array}\right) \frac{\ell !}{m_{\lambda}}$ ways to choose positions of descents, the total number of lattice paths in $L$ is

$$
\left(\begin{array}{c}
n \\
|\lambda|
\end{array}\right)\left(\begin{array}{c}
n+1 \\
\ell
\end{array}\right) \frac{\ell !}{m_{\lambda}}
$$

The number of flaws of a path $P$ in $L$ is the number of negative up steps. Then the number of flaws can vary from 0 to $|\lambda|$.

For a path $P$ with at least one flaw, we decompose $P$ into $P_{1} U P_{2}$ where $U$ is the first up step of height -1 . Let $Q$ be a path $P_{2} U P_{1}$. Then $Q$ is a path in $L$ with one less flaws than $P$. Conversely, let $Q$ be a path in $L$ with at most $|\lambda|-1$ flaws. Then one can decompose $Q$ into $Q_{1} U Q_{2}$ where $U$ is the last up step of height 0 and $P=Q_{2} U Q_{1}$ is the path in $L$ with one more flaws than $Q$. This correspondence provides a bijection between the set of path in $L$ with $k$ flaws and the set of paths in $L$ with $k-1$ flaws $(1 \leqslant k \leqslant|\lambda|)$. Thus the number of paths is independent of the number of flaws. Since the large Schröder paths are the paths in $L$ with no flaws, the proof is done.

Now we provide a combinatorial interpretation for the number of small Schröder paths which fills the missing part from the thesis of Huq [6]. It follows from the more general result about large Schröder paths with a fixed number of flat steps on the $x$-axis.

Theorem 7. The number of large Schröder path of length $2 n$ with $k$ up steps, $k$ down steps, and $n-k$ flat steps with $m-1$ flat steps on the $x$-axis is given by

$$
\frac{m}{n+1}\left(\begin{array}{c}
n-m \\
k-1
\end{array}\right)\left(\begin{array}{c}
n+k \\
k
\end{array}\right)
$$

Proof. Consider the set $L$ of all lattice paths from $(0,0)$ to $(2 n+1,1)$ with $k+1$ up steps, $k$ down steps, and $n-k$ flat steps with $m-1$ flat steps before the first up step or after the last up step. Since there are $m$ ways to put $m-1$ flat steps before the first up step and after the last up step, $\left(\begin{array}{c}n-m \\ k-1\end{array}\right)$ ways to arrange the remaining $k-1$ up steps and 
$n-k-m+1$ flat steps between the first and the last up step, and $\left(\left(\begin{array}{c}n+1 \\ k\end{array}\right)\right)=\left(\begin{array}{c}n+k \\ k\end{array}\right)$ ways to arrange $k$ down steps, the number of paths in $L$ is

$$
m\left(\begin{array}{c}
n-m \\
k-1
\end{array}\right)\left(\begin{array}{c}
n+k \\
k
\end{array}\right)
$$

If the number of flaws of a path is the number of nonpositive flat steps and nonpositive up steps, then the number of flaws can vary from 1 to $n+1$. Since the bijections used in the proof of the main theorem don't change the types of large Schröder paths, the rest of the proof is the same as the proof of Theorem 2 .

\section{References}

[1] S. H. An, S.-P. Eu, and S. Kim. Large Schröder paths by types and symmetric functions. Bull. Korean Math. Soc., 51(4):1229-1240, 2014.

[2] W. Y. C. Chen, N. Y. Li, and L. W. Shapiro. The butterfly decomposition of plane trees. Discrete Appl. Math., 155(17):2187-2201, 2007.

[3] Y.-M. Chen. The Chung-Feller theorem revisited. Discrete Math., 308(7):1328-1329, 2008.

[4] K. L. Chung and W. Feller. On fluctuations in coin-tossing. Proc. Nat. Acad. Sci. U.S.A., 35:605-608, 1949.

[5] S.-P. Eu, T.-S. Fu, and Y.-N. Yeh. Refined Chung-Feller theorems for lattice paths. J. Combin. Theory Ser. A, 112(1):143-162, 2005.

[6] A. Huq. Generalized Chung-Feller theorems for lattice paths. PhD thesis, 2009. Brandeis University.

[7] R. Johansson and S. Linusson. Pattern avoidance in alternating sign matrices. Ann. Comb., 11(3-4):471-480, 2007.

[8] M. Klazar. On abab-free and abba-free set partitions. European J. Combin., 17(1):5368, 1996.

[9] G. Kreweras. Sur les partitions non croisées d'un cycle. Discrete Math., 1(4):333-350, 1972.

[10] S.-C. Liu, Y. Wang, and Y.-N. Yeh. Chung-Feller property in view of generating functions. Electron. J. Combin., 18(1):Paper 104, 20, 2011.

[11] P. A. MacMahon. Memoir on the theory of the partitions of numbers, Part IV. Philos. Trans. Roy. Soc. London Ser. A, 209, 1909.

[12] T. V. Narayana. Cyclic permutation of lattice paths and the Chung-Feller theorem. Skand. Aktuarietidskr, 1967:23-30, 1967.

[13] B. Rhoades. Enumeration of connected Catalan objects by type. European J. Combin., 32(2):330-338, 2011.

[14] R. P. Stanley. Enumerative combinatorics. Vol. 2, volume 62 of Cambridge Studies in Advanced Mathematics. Cambridge University Press, Cambridge, 1999. With a foreword by Gian-Carlo Rota and appendix 1 by Sergey Fomin. 